\title{
Signature of circulating microRNAs in osteoarthritis
}

\author{
Christian Beyer, ${ }_{1}^{1}$ Anna Zampetaki, ${ }^{2}$ Neng-Yu Lin, ${ }^{1}$ Arnd Kleyer, ${ }^{1}$ Carlo Perricone, ${ }^{3}$ \\ Annamaria lagnocco, ${ }^{3}$ Alfiya Distler, ${ }^{1}$ Sarah R Langley, ${ }^{2}$ Kolja Gelse, ${ }^{4}$ \\ Stefan Sesselmann, ${ }^{5}$ Rolando Lorenzini, ${ }^{6}$ Andreas Niemeier, ${ }^{7}$ Bernd Swoboda, ${ }^{5}$ \\ Jörg H W Distler, ${ }^{1}$ Peter Santer, ${ }_{1}^{6}$ Georg Egger, ${ }^{6}$ Johann Willeit, ${ }^{8}$ Manuel Mayr, ${ }^{2}$ \\ Georg Schett, ${ }^{1}$ Stefan Kiechl ${ }^{8}$
}

\begin{abstract}
Handling editor Tore K Kvien
- Additional material is

published online only. To view please visit the journal online (http://dx.doi.org/10.1136/ annrheumdis-2013-204698).

For numbered affiliations see end of article.
\end{abstract}

Correspondence to Dr Christian Beyer, Department of Internal Medicine 3 and Institute of Clinical Immunology, FriedrichAlexander-University ErlangenNuremberg, Ulmenweg 18, Erlangen 91054, Germany; christian.beyer@uk-erlangen.de

Received 2 October 2013 Revised 14 January 2014 Accepted 15 January 2014

Published Online First

10 February 2014

CrossMark

To cite: Beyer C,
Zampetaki A, Lin N-Y, et al.
Ann Rheum Dis 2015;74:
e18.

\section{ABSTRACT}

Background Osteoarthritis is the most common form of arthritis and a major socioeconomic burden. Our study is the first to explore the association between serum microRNA levels and the development of severe osteoarthritis of the knee and hip joint in the general population.

Methods We followed 816 Caucasian individuals from 1995 to 2010 and assessed joint arthroplasty as a definitive outcome of severe osteoarthritis of the knee and hip. After a microarray screen, we validated 12 microRNAs by real-time PCR in the entire cohort at baseline.

Results In Cox regression analysis, three microRNAs were associated with severe knee and hip osteoarthritis. let-7e was a negative predictor for total joint arthroplasty with an adjusted HR of 0.75 (95\% Cl 0.58 to $0.96 ; p=0.021)$ when normalised to $\mathrm{U} 6$, and $0.76(95 \% \mathrm{Cl} 0.6$ to 0.97 ; $\mathrm{p}=0.026)$ after normalisation to the Ct average. miRNA454 was inversely correlated with severe knee or hip osteoarthritis with an adjusted HR of 0.77 (95\% Cl 0.61 to $0.97 ; p=0.028$ ) when normalised to U6. This correlation was lost when data were normalised to $\mathrm{Ct}$ average $(p=0.118)$. Finally, miRNA-885-5p showed a trend towards a positive relationship with arthroplasty when normalised to U6 (HR 1.24; 95\% Cl 0.95 to 1.62; $\mathrm{p}=0.107$ ) or to $\mathrm{Ct}$ average (HR 1.30; $95 \% \mathrm{Cl} 0.99$ to $1.70 ; p=0.056)$.

Conclusions Our study is the first to identify differentially expressed circulating microRNAs in osteoarthritis patients necessitating arthroplasty in a large, population-based cohort. Among these microRNAs, let-7e emerged as potential predictor for severe knee or hip osteoarthritis.

\section{INTRODUCTION}

Osteoarthritis (OA) is the most common form of arthritis and a leading cause of disability. ${ }^{1}$ In the general population, the prevalence of knee and hip OA ranges from 20 to $30 \%,{ }^{1}$ and $\mathrm{OA}$ affecting these two joints represents the major cause of lower extremity disability among older adults. ${ }^{2}$ In addition to the personal burden of the disease, Western societies invest up to $2 \%$ of their gross national product into the costs of OA resulting from loss of work productivity and increased medical costs. ${ }^{3}$ According to recent reports from the Organisation for Economic Co-operation and Development (OECD), the number of joint replacement surgeries as ultimate therapies for patients with knee and hip OA has almost doubled during the past decade (http://www.oecd-ilibrary.org).
OA is a progressive disease with a long silent period, which shows signs of cartilage degradation, mild-to-moderate synovial inflammation and altered bone structure, resulting in severe destruction and impaired function of the affected joints. ${ }^{4}$ Clinical symptoms and radiographic changes generally guide the diagnosis, with plain radiography remaining the gold standard. Radiography, however, is limited by poor sensitivity that hampers early detection of the disease and close follow-up. By contrast, sensitive and specific blood biomarkers to detect the initial stages of OA or even allow predicting the future development of the disease are not available in clinical routine. ${ }^{5}$ Many experimental biomarkers of cartilage metabolism lack sensitivity for early disease stages, specificity for OA and/ or robust validation in large clinical studies.

MicroRNAs (miRNAs) are small non-coding RNAs that regulate a broad spectrum of physiological cell processes during development and tissue homeostasis. Nascent miRNA transcripts are processed by the enzymes Drosha and Dicer to become mature miRNAs consisting of 21-25 nucleotides. Mature miRNAs associate with the RNA-induced silencing complex (RISC) to repress mRNA translation. ${ }^{6}$ Aberrant miRNA expression or dysregulation of miRNA processing has been linked to many pathologies, including cancer, cardiovascular or rheumatic disease. ${ }^{7-9}$ Since miRNA are protected from RNAse activity in the peripheral blood, miRNAs may serve as excellent biomarkers for physiological and pathological processes. ${ }^{10} 11$

Although dysregulated miRNA expression has been well documented in the osteoarthritic cartilage, ${ }^{9}{ }^{12-17}$ evidence on altered circulating miRNA levels in OA patients is very limited. ${ }^{18}{ }^{19}$ Hence, our project is the first to investigate the differential expression of circulating miRNAs in subjects with and without incident $\mathrm{OA}$ in a large prospective population-based cohort. Applying knee and hip joint replacement surgeries as definitive outcomes of severe OA, let-7e appeared to be the most promising predictor of severe OA with lower levels showing a dose-dependent association with increased frequencies of joint replacement surgeries.

\section{MATERIALS AND METHODS \\ Study subjects and sample collection}

The Bruneck study is a prospective populationbased survey that has been successfully used to 
profile circulating miRNA levels in diabetes and cardiovascular disease. ${ }^{20-28}$ The current study analysed the observation period from 1995 to 2010 with the study visit in 1995 serving as baseline (see also online supplement 1). The protocols of the Bruneck study were approved by the appropriate ethics committees, and all study subjects gave their written informed consent.

\section{Longitudinal assessment of arthroplasty between 1995 and 2010}

Localisation, date and circumstances of total hip and knee arthroplasty occurring between 1995 and 2010 were carefully recorded using three sources of information: subject self-report, medical records of the Bruneck Hospital and general practitioners, and a standardised evaluation of all radiographs ever taken on study subjects. Radiography was not routinely performed on all study participants to limit radiation exposure, but was initiated in those with symptoms suggestive of OA. Arthroplasty following bone fractures (documented by radiographs) was not considered in the current analysis. Subjects receiving arthroplasty met the criteria for the diagnosis of OA and showed OA of the hip or knee joint in at least two sequential radiographs (Kellgren-Lawrence score of 3 and 4) prior to surgery. 2930 Independent readers (RL, CP, and AK) unaware of the miRNA data assessed the radiographs. All arthroplasties were performed by a single team of experienced orthopaedic surgeons.

\section{Clinical history, physical examination and routine laboratory studies}

Clinical history, physical examination and routine laboratory studies were recorded as detailed in the online supplement 1 .

\section{Sampling strategy and miRNA analysis}

For the microarray screening, pooled serum from individuals with two or more knee/hip replacement surgeries due to OA and pooled serum from control individuals were investigated. In detail, two serum pools from 6 and 7 OA individuals and two serum pools from 6 and 7 control individuals from the 1995 baseline visit were used. Control individuals were identical in age ( \pm 1 year), sex, menopausal status and body mass index $( \pm$ $2 \mathrm{~kg} / \mathrm{m}^{2}$ ), but had not received arthroplasty prior to or during the study (figure 1). RNA extraction was performed using the miRNeasy kit (Qiagen). miRNAs were reverse-transcribed using the Megaplex Primer Pools (Human Pools A V.2.1), and expression was screened using a Human Taqman miRNA array (CardA V.2.1, Applied Biosystems, Darmstadt, Germany) covering 377 unique miRNAs. The miRNAs U6 and miRNA-45 were used as normalisation standards in the Taqman miRNA array. Comparison of these 377 miRNAs between the pools of patients with severe $\mathrm{OA}$ and the control pools revealed 12 miRNAs with differential expression. These 12 miRNAs were measured by quantitative (q)-PCR (TaqMan miRNA assays, Applied Biosystems) in the entire Bruneck Study cohort $(n=816)$ (figure 1). For the lack of generally accepted standards, all qPCR data were standardised to $\mathrm{Ct}$ average (ie, the $\mathrm{Ct}$ average of all miRNA measurements for each sample) or U6 (ie, a small non-coding RNA, which fulfilled the following criteria: detectable in all samples, low dispersion of expression levels and null association with OA status). Detailed description of the methodology and raw data of the qPCR measurements are provided in the online supplements 1 and 2 .

\section{Statistical analysis}

Data were analysed using SPSS V.18.0 and STATA V.10.1 software packages. Continuous variables were presented as means \pm $\mathrm{SD}$ or median (IQR), and dichotomous variables as numbers and percentages. We used general linear models or logistic regression models adjusted for age, sex and body mass index (BMI) to analyse differences in participant characteristics between those who had arthroplasty during follow-up and those who had not (table 1). Log $_{\mathrm{e}}$-transformed miRNAs were used for all computations to reduce the effect of extreme observations. Fold changes of individual miRNAs were calculated by dividing $\log _{\mathrm{e}}$-transformed miRNA levels in subjects with severe OA requiring joint replacement and all other. For computation of intervention rates, person-years of follow-up for each participant were accrued from the 1995 baseline until arthroplasty, age 90, death or 1 October 2010, whichever came first. Cox proportional hazard models were fitted to assess the association between miRNAs and arthroplasty due to severe OA during follow-up 1995-2010. The proportional hazard assumption was confirmed for miRNAs by testing their interaction with a function of survival time (Cox model with time-dependent covariates). When the exact date of arthroplasty was not available, we used the date of the first X-ray showing the implant as a surrogate. This may be viewed as an adequate approximation in most individuals and, if at all, errors on the conservative side. The base model was unadjusted, while multivariable models were adjusted for established predictors of severe OA (age, sex, BMI). Differential effects of miRNAs on the probability of arthroplasty in subgroups according to age and sex were tested by inclusion of appropriate interaction terms. Sensitivity analyses excluded subjects with joint arthroplasty prior to 1995. All reported p values were two-sided.

\section{miRNA relevance network inference and analysis}

Network inference algorithm was applied to evaluate global expression properties of miRNAs in OA as described previously, ${ }^{24} 26$ and the profiles were interrogated using the context likelihood of relatedness (CLR) between all possible miRNA pairs. A $10 \%$ false discovery rate (FDR) threshold was applied to the CLR networks, where the FDR was calculated using the q-value method. ${ }^{31}$

\section{RESULTS}

\section{Characteristics of the Bruneck cohort}

The Bruneck population is highly representative of the general community and shows characteristics similar to those of other Western countries (table 1 ). The mean age was $62.9 \pm 11.1$ years, $50.0 \%$ of the individuals were male and all subjects were of Caucasian origin. During the 15 -year follow-up, 67 of a total of the 816 individuals in the Bruneck cohort had one or more total joint replacement surgeries for severe knee or hip OA corresponding to an intervention rate of 6.9 per 1000 person-years. A total of 28 individuals received arthroplasty because of knee OA $(2.8$ per 1000 patient-years) and 41 for hip OA (4.1 per 1000 patientyears). Accordingly, two subjects received both hip and knee joints between 1995 and 2010. Overall 18 individuals underwent two or more arthroplasties due to severe OA during their lifetime.

Weight and BMI were significantly increased in subjects receiving arthroplasty for knee or hip OA $(\mathrm{p}=0.001$ and $\mathrm{p}=0.002$, table 1$)$. Individuals with arthroplasty showed increased circumferences of the waists and hips $(p=0.004$ and $p=0.001$, table 1$)$, but the waist-to-hip ratio did not differ compared with control subjects. This suggested that overweight rather than visceral adiposity is 


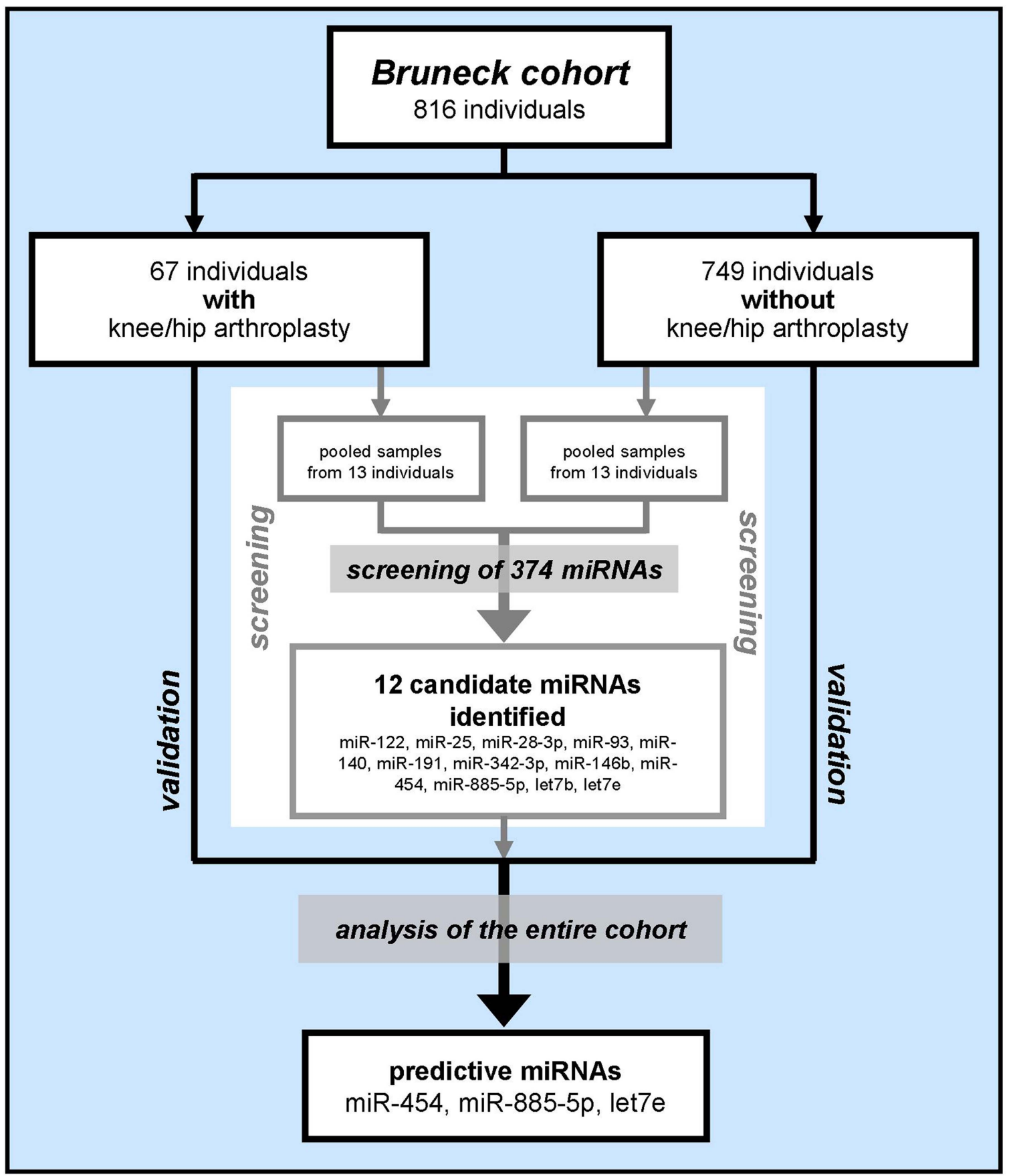

Figure 1 Study design. This study focused on the 1995-2010 follow-up period of the Bruneck cohort (1995, n=816). In this period, 67 individuals received one or more total joint replacement surgeries for severe knee or hip osteoarthritis (OA), while 749 did not. Pooled samples from 13 individuals with total joint arthroplasty versus 13 control individuals matched for sex, menopausal status, age and body mass index were screened for differential expression of miRNAs by TaqMan miRNA Array CardA for 377 unique miRNAs (screening phase: white area). The screening approach revealed 12 differentially expressed miRNAs, which were then measured by qPCR in the entire Bruneck cohort. Analyses based on these 12 miRNAs identified let-7e, miRNA-454 and miRNA-885-5p as potential predictors for severe OA of the knee and hip joints requiring arthroplasty in the general community (validation phase: grey area).

related to severe OA of the hip or knee. Moreover, there was a trend towards an increased age among individuals with arthroplasty $(p=0.053)$. By contrast, lifestyle variables and laboratory parameters did not differ between individuals with or without knee or hip arthroplasty (table 1).

\section{Circulating miRNA levels in the Bruneck study}

We were particularly interested in the predictive value of circulating miRNAs in the general population and therefore used serum samples from the 1995 baseline visit. In the initial screening, differential expression of miRNAs was assessed in pooled samples from 13 individuals with OA-related arthroplasty and 13 controls without arthroplasty matched for age, sex and BMI. Screening 377 unique miRNAs, we found 12 differentially expressed miRNA that were reliably detectable in circulation (miRNA-122, miRNA-25, miRNA 28-3p, miRNA-93, miRNA-140, miRNA-191, miRNA-342-3p, miRNA-146b, miRNA-454, miRNA-885-5p, let- 7b, let-7e). These miRNAs 
Table 1 Baseline demographic, lifestyle and laboratory characteristics in subjects with and without joint replacement surgery (1995-2010) due to severe osteoarthritis $(n=816)$

\begin{tabular}{|c|c|c|c|}
\hline \multirow[b]{2}{*}{ Characteristic* } & \multicolumn{2}{|c|}{$\begin{array}{l}\text { Joint replacement } \\
1995-2010\end{array}$} & \multirow[b]{2}{*}{$p$ Valuet } \\
\hline & No $(n=749)$ & Yes $(n=67)$ & \\
\hline Age, years & $62.7 \pm 11.3$ & $65.0 \pm 9.3$ & 0.053 \\
\hline Male sex, n (\%) & $380(50.7)$ & $28(41.8)$ & 0.161 \\
\hline Body mass index, $\mathrm{kg} / \mathrm{m}^{2}$ & $25.5 \pm 3.8$ & $27.0 \pm 4.1$ & 0.003 \\
\hline Weight, kg & $68.6 \pm 12.4$ & $72.0 \pm 13.0$ & 0.001 \\
\hline Height, $\mathrm{cm}$ & $163.7 \pm 8.9$ & $163.3 \pm 9.6$ & 0.074 \\
\hline Waist circumference, $\mathrm{cm}$ & $89.9 \pm 11.1$ & $93.6 \pm 9.8$ & 0.004 \\
\hline Hip circumference, cm & $96.6 \pm 7.8$ & $100.3 \pm 7.7$ & 0.001 \\
\hline Waist-to-hip ratio, $\mathrm{cm} / \mathrm{cm}$ & $0.92 \pm 0.07$ & $0.93 \pm 0.06$ & 0.369 \\
\hline \multicolumn{4}{|l|}{ Social status } \\
\hline Low, n (\%) & $453(60.5)$ & $44(65.7)$ & \multirow[t]{3}{*}{0.876} \\
\hline Medium, n (\%) & $165(22.0)$ & $12(17.9)$ & \\
\hline High, n (\%) & $131(17.5)$ & $11(16.4)$ & \\
\hline Fasting glucose, mg/dL & $102.0 \pm 25.0$ & $105.0 \pm 19.5$ & 0.944 \\
\hline Smoking, pack-years & $13.5 \pm 18.1$ & $9.8 \pm 14.7$ & 0.194 \\
\hline Total cholesterol, mg/dL & $229.2 \pm 42.1$ & $231.4 \pm 47.9$ & 0.960 \\
\hline HDL cholesterol, mg/dL & $58.6 \pm 16.3$ & $59.7 \pm 15.8$ & 0.199 \\
\hline LDL cholesterol, mg/dL & $144.6 \pm 37.1$ & $148.9 \pm 46.0$ & 0.661 \\
\hline Systolic blood pressure, mm Hg & $147.6 \pm 20.5$ & $154.0 \pm 21.3$ & 0.453 \\
\hline Diastolic blood pressure, $\mathrm{mm} \mathrm{Hg}$ & $86.8 \pm 9.2$ & $89.8 \pm 8.1$ & 0.236 \\
\hline Uric acid, mg/dL & $4.7 \pm 1.3$ & $4.8 \pm 1.4$ & 0.743 \\
\hline$\gamma-\mathrm{GT}, \mathrm{U} / \mathrm{L}$ & $36.7 \pm 41.5$ & $36.1 \pm 45.8$ & 0.837 \\
\hline Ferritin, $\mu \mathrm{g} / \mathrm{L}$ & $134.3 \pm 167.4$ & $143.0 \pm 162.6$ & 0.571 \\
\hline C-reactive protein, $\mathrm{mg} / \mathrm{L}$ & $1.7(0.8-3.2)$ & $1.9(0.9-3.4)$ & 0.479 \\
\hline Prior joint replacement, n (\%) & $17(2.3)$ & $1(1.5)$ & 0.507 \\
\hline \multicolumn{4}{|c|}{$\begin{array}{l}\text { *Values presented are unadjusted means } \pm \text { SD, numbers (percentages) or median } \\
\text { (IQR). Factors to convert conventional units into SI units are as follows: glucose } \\
0.05551 \text { and cholesterol } 0.02586 \text {. } \\
\text { tp Values for the variables age and sex are from unadjusted analyses and those for } \\
\text { body mass index, weight, height, waist circumference, hip circumference and WHR } \\
\text { are age-adjusted and sex-adjusted. All other p values are from general linear models } \\
\text { or logistic regression models adjusted for age, sex and body mass index. For } \\
\text { computation of p values, C-reactive protein was loge-transformed because of a } \\
\text { markedly skewed distribution. Variables in bold were statistically significant. } \\
\text { GT, glutamyl transpeptidase; HDL, high-density lipoprotein; LDL, low-density } \\
\text { lipoprotein; WHR, waist-to-hip ratio. }\end{array}$} \\
\hline
\end{tabular}

were then measured in the entire Bruneck cohort. For the lack of generally accepted standards for miRNA analysis, we normalised the miRNA expression levels to two different standards: (i) U6, a small non-coding RNA, which fulfilled the following criteria: detectable in all samples, low dispersion of expression levels and no association with OA status. (ii) $\mathrm{Ct}$ average of all tested miRNAs.

Unadjusted Cox regression analysis revealed that let-7e was inversely related to arthroplasty irrespective of the method of standardisation (normalised to either U6 or CT average) (table 2). After adjustment for sex, age and BMI, findings remained stable with an adjusted HR of 0.75 ( 0.58 to $0.95 ; \mathrm{p}=0.019)$ when normalised to $\mathrm{U} 6$, and 0.76 ( 0.60 to $0.97 ; \mathrm{p}=0.021)$ after normalisation to the $\mathrm{Ct}$ average (table 2). Findings did not change appreciably when excluding subjects with arthroplasty before 1995 (HR 0.75 (0.58 to 0.96), $\mathrm{p}=0.021$ and 0.76 ( 0.60 to 0.97$), p=0.026$ for U6 and Ct average normalised let-7e). There was no differential association between let-7e and arthroplasty in sexes and age strata. Further analysis demonstrated that the inverse association of let-7e was dose dependent. The lower the let-7e baseline levels were, the higher was the risk that individuals underwent more than one arthroplasty due to severe hip or knee OA (figure 2). Finally, our findings were similar when we analysed the relationship of let-7e with severe OA of either the hip or the knee (online supplement 1).

Besides let-7e, two other miRNAs appeared to be differentially expressed in the Bruneck cohort after adjusting for age, sex and BMI. When normalised to U6, miRNA-454 was inversely correlated with the development of OA requiring joint replacement with an adjusted $\mathrm{HR}$ of 0.77 (0.61 to 0.97; $\mathrm{p}=0.028$; table 2). Significance, however, was lost when data were normalised to the $\mathrm{Ct}$ average. A non-significant trend towards an inverse relationship between miRNA-454 and arthroplasty was observed $(\mathrm{p}=0.118)$. Similarly, miRNA-885-5p showed a non-significant trend to a positive correlation with arthroplasty after adjustment for age, sex and BMI when normalised to U6 (HR 1.24; 0.95 to $1.62 ; \mathrm{p}=0.107$ ) or the $\mathrm{Ct}$ average (HR $1.30 ; 0.99$ to $1.70 ; \mathrm{p}=0.056$; table 2 ).

Further support to the putative value of miRNA signatures as biomarkers was provided by the inference of miRNA relevance network. A differential network structure was evident between the 12 miRNAs in controls and patients with OA (FDR=10\%). The disease state was characterised by substantial edge rewiring: the positions of let-7e as well as miRNA-454 and miRNA-885-5p, the two miRNAs that were close to conventional levels of significance after adjustment for Ct average, differed substantially between individuals receiving joint arthroplasty and healthy controls, indicating a unique miRNA signature for severe OA (figure 3). In a forward stepwise selection model with a fixed entry of age, sex and BMI and allowing for all miRNAs listed in table 2, let-7e and either miRNA-454 (normalisation to $\mathrm{Ct}$ average) or miRNA-885-5p (normalisation to U6) emerged as significant risk predictors (table 3 ).

\section{DISCUSSION}

In a well-defined, population-based cohort including 816 individuals, we identified differentially expressed miRNAs as predictors for severe knee and hip OA. Applying very stringent criteria for outcome (arthroplasty) and statistical analyses (standardisation to U6 as well as Ct average), we discovered let-7e as a negative, dose-dependent predictor of OA (figure 2). Besides let-7e, weaker associations emerged for miRNA-454 and miRNA-885-5p but were not consistently significant after respective adjustments.

Patients with OA exhibit early signs of cartilage degradation, synovial inflammation and altered bone structure even before the disease becomes clinically manifest. ${ }^{4}$ Conventional radiography, which is still considered the gold standard in the diagnosis of $\mathrm{OA}$, is insensitive in detecting early disease manifestations. Magnetic resonance imaging (MRI) is emerging as a more sensitive imaging tool for OA, but is complex and expensive. In addition, MRI may not be sensitive enough to detect very early, clinically silent periods of OA. ${ }^{32}$ Thus, stable biochemical markers that are easily assessed in the peripheral blood or urine might be ideal measures to diagnose initial stages of OA, predict and assess disease progression, and monitor effects of diseasemodifying treatments. So far, a large array of biochemical markers, most of them reflecting bone and cartilage metabolism, have been studied, but few of them have shown substantial improvement in predicting $\mathrm{OA},{ }^{23}$ and none has so far entered clinical routine. Poor performance and lack of validation in large clinical trials may have been the major obstacles precluding their clinical use.

Circulating miRNAs are easily accessible and stable, ${ }^{10} 11$ which would be convenient for their daily clinical use. The stability of miRNAs is remarkable since they resist harsh 
Table 2 Association between individual microRNAs and joint replacement surgery due to severe osteoarthritis ( $\mathrm{n}=816)$ follow-up 1995-2010

\begin{tabular}{|c|c|c|c|c|c|}
\hline & \multirow{2}{*}{$\begin{array}{l}\text { Fold changes } \\
\text { to control }\end{array}$} & \multicolumn{2}{|l|}{ Unadjusted analysis } & \multicolumn{2}{|c|}{$\begin{array}{l}\text { Age-adjusted, sex-adjusted and } \\
\text { BMI-adjusted analysis }\end{array}$} \\
\hline & & HR $(95 \% \mathrm{Cl})$ & p Value & HR (95\% CI) & p Value \\
\hline \multicolumn{6}{|l|}{$\begin{array}{l}\text { microRNAs } \\
\text { normalised to U6 }\end{array}$} \\
\hline miRNA-122 & 1.36 & 1.17 (0.90 to 1.52$)$ & 0.245 & $1.10(0.84$ to 1.43$)$ & 0.499 \\
\hline miRNA-25 & 0.93 & 0.91 (0.72 to 1.14$)$ & 0.398 & $0.90(0.72$ to 1.13$)$ & 0.375 \\
\hline miRNA-28-3p & 0.56 & 0.83 (0.65 to 1.05$)$ & 0.114 & 0.85 (0.67 to 1.07$)$ & 0.166 \\
\hline miRNA-93 & 0.99 & $0.98(0.77$ to 1.23$)$ & 0.834 & $0.98(0.77$ to 1.24$)$ & 0.833 \\
\hline miRNA-140 & 0.95 & $0.96(0.76$ to 1.21$)$ & 0.717 & $0.95(0.75$ to 1.21$)$ & 0.668 \\
\hline miRNA-191 & 0.92 & $0.86(0.67$ to 1.10$)$ & 0.223 & 0.91 (0.71 to 1.17$)$ & 0.465 \\
\hline miRNA-342-3p & 0.93 & 0.87 (0.60 to 1.09$)$ & 0.231 & $0.89(0.70$ to 1.13$)$ & 0.333 \\
\hline miRNA-146b & 0.96 & $0.90(0.71$ to 1.15$)$ & 0.402 & $0.92(0.72$ to 1.17$)$ & 0.489 \\
\hline miRNA-454 & 0.85 & 0.86 (0.67 to 1.09$)$ & 0.206 & $0.77(0.61$ to 0.97$)$ & 0.028 \\
\hline miRNA-885-5p & 1.12 & $1.18(0.91$ to 1.54$)$ & 0.213 & 1.24 (0.95 to 1.62$)$ & 0.107 \\
\hline miRNA-let-7b & 0.87 & 0.91 (0.72 to 1.15$)$ & 0.426 & $0.93(0.73$ to 1.20$)$ & 0.579 \\
\hline miRNA-let-7e & 0.66 & $0.72(0.57$ to 0.92$)$ & 0.007 & $0.75(0.58$ to 0.95$)$ & 0.019 \\
\hline \multicolumn{6}{|c|}{$\begin{array}{l}\text { microRNAs } \\
\text { normalised to Ct average }\end{array}$} \\
\hline miRNA-122 & 1.63 & 1.25 (0.95 to 1.64$)$ & 0.119 & $1.15(0.87$ to 1.51$)$ & 0.341 \\
\hline miRNA-25 & 0.89 & $0.92(0.74$ to 1.15$)$ & 0.464 & $0.91(0.74$ to 1.14$)$ & 0.416 \\
\hline miRNA-28-3p & 0.91 & 0.80 (0.63 to 1.01$)$ & 0.055 & 0.83 (0.66 to 1.05$)$ & 0.121 \\
\hline miRNA-93 & 1.08 & 1.03 (0.81 to 1.32$)$ & 0.784 & 1.03 (0.81 to 1.32$)$ & 0.804 \\
\hline miRNA-140 & 1.08 & 1.03 (0.81 to 1.31$)$ & 0.835 & 1.01 (0.79 to 1.29 ) & 0.963 \\
\hline miRNA-191 & 0.95 & 0.88 (0.69 to 1.11$)$ & 0.267 & 0.97 (0.76 to 1.23$)$ & 0.778 \\
\hline miRNA-342-3p & 0.88 & 0.89 (0.71 to 1.13$)$ & 0.336 & 0.91 (0.71 to 1.17$)$ & 0.475 \\
\hline miRNA-146b & 0.92 & $1.00(0.79$ to 1.28$)$ & 0.974 & $1.01(0.80$ to 1.29$)$ & 0.926 \\
\hline miRNA-454 & 0.80 & 0.95 (0.75 to 1.21$)$ & 0.686 & $0.83(0.65$ to 1.04$)$ & 0.118 \\
\hline miRNA-885-5p & 2.03 & $1.24(0.95$ to 1.60$)$ & 0.110 & $1.30(0.99$ to 1.70$)$ & 0.056 \\
\hline miRNA-let7b & 0.87 & 0.92 (0.73 to 1.17$)$ & 0.504 & 0.97 (0.76 to 1.23$)$ & 0.773 \\
\hline miRNA-let-7e & 0.45 & 0.71 (0.56 to 0.90$)$ & 0.004 & $0.76(0.60$ to 0.96$)$ & 0.021 \\
\hline
\end{tabular}

conditions that would normally degrade most RNAs, including boiling, extreme $\mathrm{pH}$, long-time storage and repeated freezethaw cycles. ${ }^{33}$ Our data obtained in a large cohort representative of the general community are the first to raise evidence that specific miRNAs, such as let-7e, could indeed serve as a valuable biomarker for OA. Despite the large size of our cohort, however, the number of joint replacements was comparatively low and further studies need to confirm and validate our findings in other cohorts and ethnicities. Our study was designed to identify candidate miRNA to predict severe OA; thus, the validity of let-7e, miRNA-454 and miRNA-885-5p for early detection and follow-up remains to be addressed.
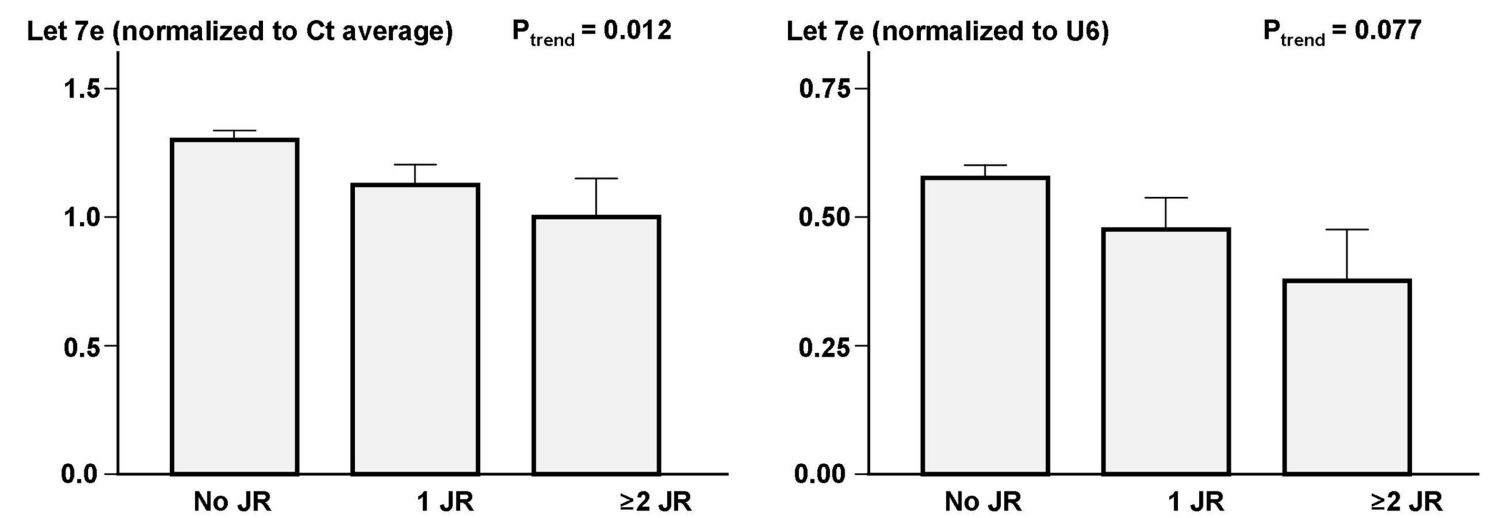

Figure 2 Baseline levels of miRNA-let-7e according to the number of surgical joint replacements (JR) due to severe osteoarthritis. This figure indicates a dose-response relation. Data are presented separately for miRNA levels normalised to U6 and the Ct average of all miRNAs assessed. Values are unadjusted geometric means+SE. Numbers of subjects in the groups with no JR, one JR and two or more JR were 732, 66 and 18. 

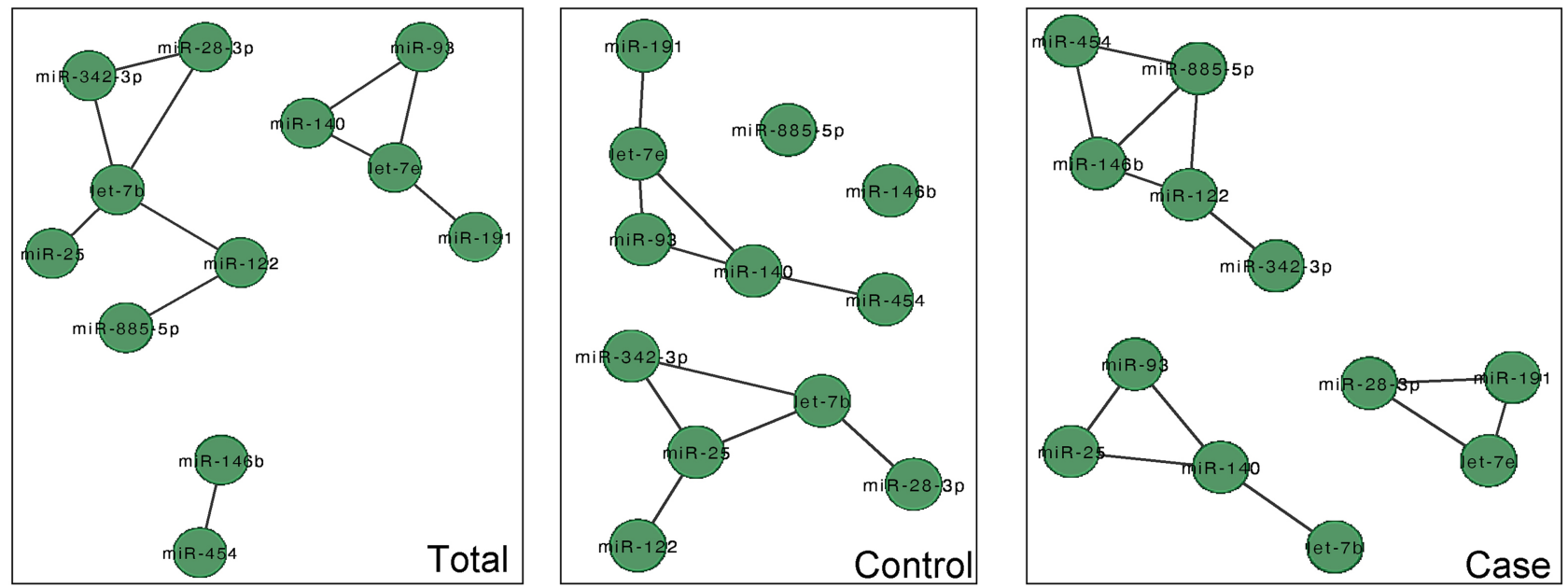

FDR 10\%

Figure 3 miRNA relevance network inference and analysis. Differential network structure between 12 miRNAs in controls and osteoarthritis cases. Nodes represent individual miRNAs and edges (links) represent the extent of expression similarity measured using the context likelihood of relatedness algorithm.

While our study is the first to investigate the levels of circulating miRNAs within a large population-based cohort, several studies have assessed differential miRNA expression in the cartilage of OA patients. ${ }^{9}{ }^{12-17}$ None of the miRNAs that have already been associated with physiological or pathological cartilage metabolism, however, was differentially expressed in the OA patients of our cohort. Several reasons might account for these differences: small study cohorts and differences in the methodology of collecting articular cartilage may lead to differences in miRNA expression profiles. Patient selection and different cohorts (early vs late disease, mild vs severe disease) might also

Table 3 Multivariable risk predictors of joint replacement surgery due to severe osteoarthritis ( $n=816$ ) follow-up 1995-2010

\begin{tabular}{llll}
\hline Variables & $\begin{array}{l}\text { Step of } \\
\text { entry }\end{array}$ & $\begin{array}{l}\text { HR (95\% Cl) per } 1 \text { SD } \\
\text { unit increase in variable } \\
\text { levels* }\end{array}$ & p Value \\
\hline $\begin{array}{l}\text { microRNAs } \\
\text { normalised to U6 }\end{array}$ & & $1.85(1.39$ to 2.45$)$ & \\
$\quad$ Age & $0.79(0.48$ to 1.29$)$ & $<0.001$ \\
Male sex & & $1.31(1.04$ to 1.64$)$ & 0.340 \\
Body mass index & & $0.70(0.54$ to 0.89$)$ & 0.021 \\
$\quad$ miRNA-let-7e & 1 & $1.35(1.04$ to 1.77$)$ & 0.005 \\
$\quad$ miRNA-885-5p & 2 & & 0.025 \\
microRNAs & & $1.93(1.45$ to 2.57$)$ & \\
normalised to Ct average & & $0.84(0.51$ to 1.37$)$ & $<0.001$ \\
Age & & $1.31(1.05$ to 1.65$)$ & 0.472 \\
Male sex & $0.70(0.55$ to 0.88$)$ & 0.017 \\
Body mass index & & $0.73(0.56$ to 0.94$)$ & 0.003 \\
$\quad$ miRNA-let-7e & 1 & & 0.017 \\
$\quad$ miRNA-454 & 2 &
\end{tabular}

$\mathrm{HRs}, 95 \% \mathrm{Cl}$ and $\mathrm{p}$ values were derived from multivariable Cox regression analysis. Variables were selected for inclusion from all miRNAs listed in table 2 using a forward stepwise selection procedure with a fixed entry of age, sex and body mass index.

${ }^{*}$ To facilitate a comparison between the various risk predictors, HRs were calculated for a 1 SD unit increase in metric variables. contribute to the observed differences. In this context, even the studies that focused on the miRNA expression in articular cartilage reported striking differences in their miRNA profiles. Apart from that, it is still unclear whether or not the circulating miRNA signature directly emerges from the articular cartilage or other tissues. While elegant studies from the cancer field suggest that differentially expressed miRNAs could indeed emerge from the pathological tissues, ${ }^{10}{ }^{34}$ we have preliminary data that challenge this concept in OA. In cartilage from patients receiving hip replacement surgery due to severe OA compared with patients receiving hip arthroplasty for femoral fracture, we did not observe a differential expression of our serum miRNA candidates. In line with our observations, a small study demonstrated that miRNA expression profiles in the synovial fluid differed from miRNA signatures in the serum of the matched OA patients. ${ }^{18}$ Despite limitations in cohort size and miRNA selection, these findings suggest that it may well be that miRNA signatures found in $\mathrm{OA}$ patients do not stem from the non-vascularised, bradytrophic cartilage but from other, much better vascularised tissues, such as the synovia, the bone or the bone marrow.

Taken together, we identified differentially expressed circulating miRNAs in patients with severe OA in a large, populationbased cohort. let-7e, and possibly miRNA-454 and miRNA-885-5p, may represent predictors for severe knee or hip OA necessitating arthroplasty and could thus be further developed into predictive biomarkers for OA. let-7e emerged as the most promising candidate to predict the OA risk since its association to OA was independent of age, sex and BMI, and consistent in subgroups and with different standardisation procedures. Moreover, there was a clear dose-response relationship (figure 2). Technical innovations, including small disposable microchips to assess OA-specific miRNAs, might foster the clinical application of this technology. Prior to a potential clinical use, however, further studies are needed to validate the candidate miRNAs in different cohorts and for different applications, such as diagnosis, risk prediction and disease activity, to assess their incremental value beyond the information provided by standard risk predictors. 


\section{Author affiliations}

${ }^{1}$ Department of Internal Medicine 3 and Institute of Clinical Immunology, University Erlangen-Nuremberg, Erlangen, Germany

2King's British Heart Foundation Centre, King's College London, London, UK

${ }^{3}$ Rheumatology, Department of Internal Medicine and Medical Specialties, Sapienza University of Rome, Rome, Italy

${ }^{4}$ Department of Trauma Surgery, University Erlangen-Nuremberg, Erlangen, Germany ${ }^{5}$ Department of Orthopedic Surgery, University Erlangen-Nuremberg, Erlangen, Germany

${ }^{6}$ Departments of Radiology and Internal and Laboratory Medicine, Bruneck Hospital, Bruneck, Italy

${ }^{7}$ Department of Orthopaedics, University Medical Center Hamburg-Eppendorf, Hamburg, Germany

${ }^{8}$ Department of Neurology, Medical University of Innsbruck, Innsbruck, Austria

Correction notice This article has been corrected since it was published Online First. The funding section has been amended.

Contributors JW, MM, GS and SK conceived and designed the experiments. $C B$, $A Z, N-Y L, A K, C P, A D, S L, K G, S S, R L, A N, B S, J H W D$, PS and GE performed the experiments. CB, AZ, SL, JW, MM, GS and SK analysed the data. CB, JW, MM, GS and SK wrote the first draft of the manuscript. All authors contributed to the writing of the manuscript.

Funding This work was supported by the Department of Health via the National Institute for Health Research (NIHR) Biomedical Research Centre based at Guy's and St Thomas' NHS Foundation Trust and King's College London, in partnership with King's College Hospital NHS Foundation Trust. M.M. is a Senior Fellow of the British Heart Foundation. The Bruneck Study was supported by the "Pustertaler Verein zur Prävention von Herz- und Hirngefässerkrankungen", the "Gesundheitsbezirk Bruneck" and the "Assessorat für Gesundheit und Sozialwesen", Bolzano, Italy. The funders had no role in study design, data collection and analysis, decision to publish, or preparation of the manuscript. Individual funding: CB: Erlanger Leistungsbezogene Anschubfinanzierung und Nachwuchsförderung (project: 53410037), Grant J29 of the Interdisciplinary Center of Clinical Research (IZKF) Erlangen and Grant BE 5191/1-1 of the Deutsche Forschungsgemeinschaft. CB and SS: Erlanger Leistungsbezogene Anschubfinanzierung und Nachwuchsförderung (project: 53410034).

\section{Competing interests None.}

Patient consent Obtained.

Ethics approval Regional ethics committee.

Provenance and peer review Not commissioned; externally peer reviewed.

\section{REFERENCES}

1 Lawrence RC, Felson DT, Helmick CG, et al. Estimates of the prevalence of arthritis and other rheumatic conditions in the United States. Part II. Arthritis Rheum 2008;58:26-35.

2 Guccione AA, Felson DT, Anderson JJ, et al. The effects of specific medical conditions on the functional limitations of elders in the Framingham Study. Am J Public Health 1994;84:351-8.

3 Reginster JY. The prevalence and burden of arthritis. Rheumatology (Oxford) 2002;41(Supp 1):3-6.

4 Goldring MB, Goldring SR. Articular cartilage and subchondral bone in the pathogenesis of osteoarthritis. Ann N Y Acad Sci 2010;1192:230-7.

5 Patra D, Sandell $\amalg$. Recent advances in biomarkers in osteoarthritis. Curr Opin Rheumatol 2011;23:465-70.

6 He L, Hannon GJ. MicroRNAs: small RNAs with a big role in gene regulation. Nat Rev Genet 2004:5:522-31.

7 Esteller M. Non-coding RNAs in human disease. Nat Rev Genet 2011;12:861-74.

8 Goldring MB, Marcu KB. Epigenomic and microRNA-mediated regulation in cartilage development, homeostasis, and osteoarthritis. Trends $\mathrm{Mol} \mathrm{Med}$ 2012;18:109-18

9 Le LT, Swingler TE, Clark IM. Review: The Role of MicroRNAs in Osteoarthritis and Chondrogenesis. Arthritis Rheum 2013;65:1963-74.
10 Mitchell PS, Parkin RK, Kroh EM, et al. Circulating microRNAs as stable blood-based markers for cancer detection. Proc Natl Acad Sci U S A 2008; 105:10513-18.

11 Wang K, Zhang S, Marzolf B, et al. Circulating microRNAs, potential biomarkers for drug-induced liver injury. Proc Natl Acad Sci U S A 2009;106:4402-7.

12 Araldi E, Schipani E. MicroRNA-140 and the silencing of osteoarthritis. Genes Dev 2010;24:1075-80.

13 lliopoulos D, Malizos KN, Oikonomou P, et al. Integrative microRNA and proteomic approaches identify novel osteoarthritis genes and their collaborative metabolic and inflammatory networks. PLOS ONE 2008;3:e3740.

14 Li X, Gibson G, Kim JS, et al. MicroRNA-146a is linked to pain-related pathophysiology of osteoarthritis. Gene 2011;480:34-41.

15 Miyaki S, Nakasa T, Otsuki S, et al. MicroRNA-140 is expressed in differentiated human articular chondrocytes and modulates interleukin-1 responses. Arthritis Rheum 2009;60:2723-30.

16 Miyaki S, Sato T, Inoue A, et al. MicroRNA-140 plays dual roles in both cartilage development and homeostasis. Genes Dev 2010;24:1173-85.

17 Yamasaki K, Nakasa T, Miyaki S, et al. Expression of MicroRNA-146a in osteoarthritis cartilage. Arthritis Rheum 2009;60:1035-41.

18 Murata K, Yoshitomi H, Tanida S, et al. Plasma and synovial fluid microRNAs as potential biomarkers of rheumatoid arthritis and osteoarthritis. Arthritis Res Ther 2010;12:R86

19 Murata $\mathrm{K}$, Furu M, Yoshitomi $\mathrm{H}$, et al. Comprehensive microRNA analysis identifies miRNA-24 and miRNA-125a-5p as plasma biomarkers for rheumatoid arthritis. PLoS One 2013;8:e69118.

20 Kiechl S, Lorenz E, Reindl M, et al. Toll-like receptor 4 polymorphisms and atherogenesis. N Engl I Med 2002;347:185-92.

21 Kiechl S, Schett G, Schwaiger J, et al. Soluble receptor activator of nuclear factor-kappa B ligand and risk for cardiovascular disease. Circulation 2007;116:385-91.

22 Schett $\mathrm{G}$, Kiechl $\mathrm{S}$, Redlich $\mathrm{K}$, et al. Soluble RANKL and risk of nontraumatic fracture. JAMA 2004:291:1108-13.

23 Schett G, Kiechl S, Bonora E, et al. Vascular cell adhesion molecule 1 as a predictor of severe osteoarthritis of the hip and knee joints. Arthritis Rheum 2009;60:2381-9.

24 Zampetaki A, Kiechl S, Drozdov I, et al. Plasma microRNA profiling reveals loss of endothelial miRNA-126 and other microRNAs in type 2 diabetes. Circ Res 2010;107:810-17.

25 Kiechl S, Schett G, Wenning G, et al. Osteoprotegerin is a risk factor for progressive atherosclerosis and cardiovascular disease. Circulation 2004; 109:2175-80.

26 Zampetaki A, Willeit $\mathrm{P}$, Tilling L, et al. Prospective Study on Circulating MicroRNAs and Risk of Myocardial Infarction. J Am Coll Cardiol 2012;60:290-9.

27 Willeit P, Zampetaki A, Dudek K, et al. Circulating MicroRNAs as Novel Biomarkers for Platelet Activation. Circ Res 2013;112:595-600.

28 Kiechl S, Wittmann J, Giaccari A, et al. Blockade of receptor activator of nuclear factor-kappaB (RANKL) signaling improves hepatic insulin resistance and prevents development of diabetes mellitus. Nat Med 2013;19:358-63.

29 Altman R, Alarcon G, Appelrouth D, et al. The American College of Rheumatology criteria for the classification and reporting of osteoarthritis of the hip. Arthritis Rheum 1991;34:505-14.

30 Altman R, Asch E, Bloch D, et al. Development of criteria for the classification and reporting of osteoarthritis. Classification of osteoarthritis of the knee. Diagnostic and Therapeutic Criteria Committee of the American Rheumatism Association. Arthritis Rheum 1986:29:1039-49.

31 Storey JD. A direct approach to false discovery rates. J R Statist Soc 2002:64:479-98.

32 Menashe L, Hirko K, Losina E, et al. The diagnostic performance of MRI in osteoarthritis: a systematic review and meta-analysis. Osteoarthritis Cartilage 2012;20:13-21.

33 Cortez MA, Bueso-Ramos C, Ferdin J, et al. MicroRNAs in body fluids--the mix of hormones and biomarkers. Nat Rev Clin Oncol 2011;8:467-77.

34 Chen $X, B a Y, M a L$, et al. Characterization of microRNAs in serum: a novel class of biomarkers for diagnosis of cancer and other diseases. Cell Res 2008; 18:997-1006. 


\section{ARD Signature of circulating microRNAs in osteoarthritis}

Christian Beyer, Anna Zampetaki, Neng-Yu Lin, Arnd Kleyer, Carlo

Perricone, Annamaria lagnocco, Alfiya Distler, Sarah R Langley, Kolja Gelse, Stefan Sesselmann, Rolando Lorenzini, Andreas Niemeier, Bernd Swoboda, Jörg H W Distler, Peter Santer, Georg Egger, Johann Willeit, Manuel Mayr, Georg Schett and Stefan Kiechl

Ann Rheum Dis 2015 74: e18 originally published online February 10, 2014

doi: 10.1136/annrheumdis-2013-204698

Updated information and services can be found at:

http://ard.bmj.com/content/74/3/e18

These include:

Supplementary Supplementary material can be found at:

Material http://ard.bmj.com/content/suppl/2014/02/10/annrheumdis-2013-2046 98.DC1

References This article cites 34 articles, 8 of which you can access for free at: http://ard.bmj.com/content/74/3/e18\#BIBL

Email alerting Receive free email alerts when new articles cite this article. Sign up in the service box at the top right corner of the online article.

Topic Articles on similar topics can be found in the following collections Collections

\section{Notes}

To request permissions go to:

http://group.bmj.com/group/rights-licensing/permissions

To order reprints go to:

http://journals.bmj.com/cgi/reprintform

To subscribe to BMJ go to:

http://group.bmj.com/subscribe/ 